\title{
Low-mass star formation in bright rimmed clouds
}

\author{
Migenes, V. ${ }^{1}$, Trinidad, M. A. ${ }^{1}$, \\ Valdettaro, R. ${ }^{2}$, Palla, F. ${ }^{2}$ and Brand, J. ${ }^{3}$ \\ ${ }^{1}$ Department of Astronomy, University of Guanajuato, P.O. Box 144, Guanajuato, \\ GTO. Mexico 36000 \\ email: vmigenes@astro.ugto.mx, trinidad@astro.ugto.mx \\ ${ }^{2}$ Osservatorio Astrofisico di Arcetri, Largo Enrico Fermi 5, Firenze, Italy 50125 \\ email: rv@arcetri.astro.it, email: palla@arcetri.astro.it \\ ${ }^{3}$ Istituto di Radioastronomia, Via Gobetti 101, Bologna, Italy I-40129 \\ email: brand@ira.cnr.it
}

\begin{abstract}
Bright Rimmed Clouds (BRCs) are clouds that have been compressed by an external ionization-shock front which focuses the neutral gas into compact globules. The boundary layer between the neutral gas and the gas ionized by the incident photons is often called "bright rim" but the clumps are sometimes classified also as speck globules or cometary globules depending on their appearance. Small globules with bright rims have been considered to be potential sites of star formation and have been studied in several individual regions. We present the first high resolution VLA observations of 20 of these BRCs, but only three detections were obtained. The low detection rate seems to support the idea that BRCs produce mostly low-luminosity objects, for which maser emission is weak and episodic, and that the embedded sources are in a more advanced evolutionary phase than class 0 objects.
\end{abstract}

Keywords. ISM: clouds, star: formation, masers

\section{Observations}

The observations were made with the VLA of the National Radio Astronomy Observatory (NRAO) in its B configuration during four runs (approx 2 hours long each) on 2005 February 20, 24, 25 and March 30. We observed water maser emission, in LCP mode, during 15 minutes on each target source. We used a bandwidth of $3.125 \mathrm{MHz}$ with 127 channels, giving a velocity resolution of $0.33 \mathrm{~km} \mathrm{~s}^{-1}$, and a velocity range of $\sim 40 \mathrm{~km}$ $\mathrm{s}^{-1}$. The data were reduced with standard techniques using the NRAO AIPS software package.

Though all 44 northern BRCs, from the list of Sugitani et al (1991), have been observed by Valdettaro et al. (2005) only twenty were observed with the VLA. Only three were detected, all towards the HII region IC1396, also known as S131, in the Cep OB2 association at a distance of $750 \mathrm{pc}$. IC $1396 \mathrm{~N}$ is an extended HII region ( 3deg in diameter) excited by the O6 star HD 206267 and a cluster of B stars. This region is associated with $\mathrm{OH}$ and $\mathrm{H}_{2} \mathrm{O}$ maser emission (Slysh et al. 1999 and Migenes et al. 1999) in addition to other molecular species.

\section{Discussion: The detected sources}

The IRAS source associated with this globule, IRAS 21346+5714 (BRC36 from Sugitani's catalog), has a luminosity of $110 L_{\odot}$ and FIR-colors typical of protostellar candidates or precursors to UCHII regions. It is located in the tail of the globule, rather than 
Table 1. Detected Sources

\begin{tabular}{lccrrr}
\hline Name & $\begin{array}{c}R A(J 2000) \\
(\text { hms })\end{array}$ & $\begin{array}{c}\text { DEC(J2000) } \\
\left(d^{\prime *}\right)\end{array}$ & $\begin{array}{r}\text { Flux } \\
(\text { Jy/b })\end{array}$ & $\begin{array}{r}\mathrm{V}_{L S R} \\
(\mathrm{~km} / \mathrm{s})\end{array}$ \\
\hline IRAS 21346+5714 & 213607.881 & +572643.43 & 32.32 & -10.3 \\
IRAS 21388+5622 & 214028.107 & +5636 & 02.16 & 0.28 & -3.0 \\
IRAS 21445+5712 & 2146 & 47.140 & +572630.85 & 0.17 & -6.1 \\
\hline
\end{tabular}

in its head, close to the bright rim as in most BRCs. Single-dish monitoring observations (Valdettaro et al. 2005) show very variable $\mathrm{H}_{2} \mathrm{O}$ maser emission, which is common in lowluminosity sources. The VLA observations reveal a single spectral component at about $-10.4 \mathrm{~km} \mathrm{~s}^{-1}$. The map shows a spot with extended E-W structure, about $2 \mathrm{~km} \mathrm{~s}^{-1}$ wide, suggesting that higher resolution may show more than one component.

The globule associated with IRAS 21388+5622 (BRC37 from Sugitani's catalog) has a cometary rim morphology. The embedded IRAS source has a luminosity of $110 L_{\odot}$ (Sugitani et al. 1991), classified as a Class 0 like object by Sugitani et el. (2000) on the basis of the ratio $L_{b o l} / L_{s u b}$. The IRAS source is also associated with the densest core of the whole globule. Duvert et al. (1990) detected a bipolar outflow with a 20" separation between the red and blue lobes and a N-S orientation. The VLA spectrum exhibits 2 features that the map reveals as 3 separate spots $1.0 \mathrm{~km} \mathrm{~s}^{-1}$ wide.

The source IRAS 21445+5712 (BRC39 from Sugitani's catalog) is an embedded IRAS source with a luminosity of $116 L_{\odot}$. The globule has a tightly curved rim morphology and located near the center of the BRC (Schwartz et al. 1991). The VLA position of the $\mathrm{H}_{2} \mathrm{O}$ maser is quite close to that of the IRAS source, indicating that the latter is the exciting source. Froebrich et al. (2005) found in the optical field a new giant flow emerging from the embedded IRAS source. These observations show a single-feature spectrum and the map presents a single spot $1.6 \mathrm{~km} \mathrm{~s}^{-1}$ wide.

\section{Conclusions}

The low detection rate $(3 / 20)$ for water vapor maser emission in BRCs, is not completely due to the sensitivity of the observations, but it could also be due to lower FIR flux from the YSO than what is detected by IRAS. The warm dust within and close to the bright rim strongly contributes to its flux as measured by IRAS. The much lower intrinsic FIR flux of the YSO would suggest that BRCs are sites of low-mass-star formation. BRCs seem to produce mostly low-luminosity objects, which are presently in a more advance evolutionary phase than class 0 sources, for which the frequency of occurrence of maser emission is low and highly episodic. The high resolution positions of the water vapor maser emission will help us identify their exciting source.

\section{References}

Duvert, G. et al. 1990, A\&A 233, 190

Froebrich, D. et al. 2005, A\&\&A 432, 575

Migenes V., et al. 1999, ApJS 123, 487

Slysh, V. I. et al. 1999, ApJ 526, 238

Sugitani, K., Fukui, Y. \& Ogura, K., 1991, ApJS 77, 59

Sugitani, K. et al. 2000, AJ 119, 323

Valdettaro, R. et al. 2005, A\&A 443, 535

Schwartz, R. D., Wilking, B. A., \& Giulbudagian, A. L., 1991, ApJ 370, 262 